\title{
EL TRABAJO PRÁCTICO EN LA ENSEÑANZA DE LAS CIENCIAS: UNA REVISIÓN
}

\author{
BARBERÁ, O. ${ }^{1}$ y VALDÉS, P. ${ }^{2}$ \\ 1 Departament de Didàctica de Jes Ciències de la Universitat de València. Ap. correus 22045. \\ E-46071 València. \\ 2 Instituto Superior Pedagógico Enrique J. Varona. 11400 Ciudad de La Habana, Cuba.
}

\begin{abstract}
SUMMARY
This article is a review about the effectiveness of practicals in science education at the secondary and tertiary levels. Through more than one hundred references, the authors conclude solid arguments have not been provided to justify the great deal of material and human resources needed for the implementation of this educational tool. In spite of these disappointing findings, the authors do not deny the central role of practicals in science education, and they justify these negative results by invoking the great complexity of the subject, the ambiguity that surrounds the term "practicals", and an unsuitable methodology that researchers use to measure their effectiveness. Finally, some plausible solutions to the problem are suggested, such as the characterisation of the cducational objectives of practicals, or the use of a qualitative evaluation methodology.
\end{abstract}

\section{INTRODUCCIÓN}

Sin duda, el trabajo práctico y, en particular, la actividad de laboratorio constituye un hecho diferencial propio de la enseñanza de las ciencias. Hace casi trescientos años que John Locke propuso la necesidad de que los estudiantes realizaran trabajo práctico en su educación, y a finales del siglo XIX ya formaba parte integral del currículo de ciencias en Inglaterra y Estados Unidos (Gee y Clackson, 1992; Layton, 1990; Lock, 1988). Desde entonces, se ha mantenido una fe inamovible en la tradición que asume la gran importancia del trabajo práctico para la enseñanza de las ciencias.

No obstante, esta creencia en la utilidad del trabajo práctico también ha tenido sus críticas desde antiguo. Ya en 1892 se recogen testimonios de ellas: Hace unos pocos años se urgia a los profesores a adoptar los métodos de laboratorio para ilustrar los libros de texto; ahora parece al menos tan necesario urgirlos a utilizar el libro de texto para hacer inteligible el caótico trabajo de laboratorio (Moyer, 1976).
La mayoría de la investigación realizada para estudiar la efectividad del trabajo práctico en la enseñanza de las ciencias no ha mostrado resultados concluyentes, y el papel que éste ha ocupado en los currículos de ciencias en el transcurso de nuestro siglo ha sido siempre objeto de controversia. En los años sesenta, proyectos en Estados Unidos como Biological Sciences Curriculum Study (BSCS), Chemical Education Material Study (CHEM Study) o Physical Science Study Committee (PSSC), así como los cursos Nuffield de biología, física y química en Inglaterra, realizaron una fuerte promoción de un estilo de enseñanza que suponía que el trabajo práctico realizado por los alumnos les conduciría a los fundamentos conceptuales, ocupando el profesor un papel de apoyo y guía para que los alumnos descubriesen los nuevos conceptos (Mayer, 1986). Desde entonces, los profesores hemos considerado el trabajo práctico como una estrategia educativa útil para conseguir casi cualquier objetivo educativo planteado; sin embargo, con frecuencia nosotros mismos somos incapaces de manifestar 
claramente el papel y los objetivos que esperamos de é. Además, se puede decir que las investigaciones que se han dedicado a comprobar su eficiencia, en su mayoría, concluyen que los alumnos han obtenido poco, si algún, beneficio del trabajo práctico realizado (Clakson y Wright, 1992).

Así, aunque el trabajo práctico es habitualmente considerado inestimable en la enseñanza de las ciencias, la investigación parece mostrar que no siempre resulta tan valioso para su aprendizaje. Si bien, para muchos, la educación científica se queda incompleta sin haber obtenido alguna experiencia en el laboratorio, no es menos cierto que el trabajo práctico no es una panacea universal en la enseñanza de las ciencias para conseguir cualquier objetivo educativo. De hecho, si comparamos con el entusiasmo existente en los años sesenta, ahora hay menos confianza entre los investigadores en que el uso del laboratorio y del trabajo de campo ayude realmente a los alumnos a mejorar la adquisición de conceptos científicos (Buchan y Jenkins, 1992; Thijs y Bosch, 1995). No obstante, profesores, diseñadores curriculares, administradores educativos, padres, gobiernos siguen apostando con su esfuerzo y con su dinero por el trabajo práctico, convencidos de que éste añade una dimensión especial a la ensef̃anza de las ciencias que va más allá de lo que se puede obtener escuchando las explicaciones de un profesor u observando sus demostraciones en el laboratorio. $Y$ es tiempo de que averigitemos si existe realmente esa dimensión especial y si podemos proporcionar una razón sólida para justificar el esfuerzo que supone la realización del trabajo práctico en la enseñanza.

\section{¿QUÉ SE ESPERA DEL TRABAJO PRACTICO?}

Desafortunadamente, no hay consenso sobre los objetivos del trabajo de laboratorio, ní sobre su aportación específica a la educación científica. Desde un punto de vista constructivista, un papel atractivo para las prácticas sería su capacidad de promover el cambio conceptual, que las experiencias en el laboratorio proporcionaran a los alumnos la oportunidad de cambiar sus creencias superficiales por enfoques científicos más sofisticados sobre los fenómenos naturales. Esto no parece que sea tan sencillo, y tanto la práctica docente como algunos resultados de investigación proporcionan evidencias convincentes de que los preconceptos persisten incluso cuando son enfrentados a la experiencia directa que los contradice (Driver, 1985; Watson et al., 1995; White, 1991)

En una revisión bibliográfica que cubre los años sesenta, ya se constata una gran disparidad entre los objetivos que proponen para el trabajo práctico investigadores, diseñadores curriculares, profesores y alumnos en los distintos niveles educativos. Mientras que profesores e investigadores proponen como objetivos principales el descubrir las leyes a través de la experiencia, el adiestrar a los estudiantes en la realización de informes experimentales y en la confección de un diario de laboratorio, o el servir de motivación para mantener el interés en el estudio de las ciencias, la visión de los estudiantes acerca del papel que cumple el trabajo práctico en su instrucción es bastante diferente. Según los resultados del informe de 1969 de la Comisión sobre la Enseñanza en la Educación Superior del Sindicato Nacional de Estudiantes de Inglaterra, los objetivos del trabajo práctico son el aprendizaje de técnicas experimentales y el refuerzo de las clases teóricas, objetivos que los profesores consideran poco prioritarios. Los estudiantes también dan gran importancia a la oportunidad que proporciona el trabajo de laboratorio para la iniciativa personal y la capacidad de juzgar la calidad de un diseño experimental, así como para mantener un contacto menos formal y más estrecho con los docentes (Tremlett, 1972).

Sin embargo, una investigación similar llevada a cabo unos pocos años antes también en Inglaterra obtuvo resultados distintos. Según ella, los profesores damos a las prácticas el papel de instrumento motivador hacia las ciencias para los alumnos más jóvenes de la educación secundaria, mientras que para los más mayores las utilizamos con la esperanza de que desarrollen en ellos capacidades que les serán útiles en la mejora del aprendizaje de las ciencias en la educación superior. Los objetivos que señalaron los profesores como más importantes para el trabajo práctico fueron: el desarrollo de destrezas manipulativas, la ayuda que pueden proporcionar a la comprensión de los principios teóricos de las disciplinas y la recopilación de hechos y datos que, seguin ellos, permite el posterior descubrimiento de los principios. Sin embargo, el objetivo principal para los estudiantes según esta investigación fue la promocion del interés y la toma de contacto con la realidad de los fenómenos naturales, objetivos que Ios profesores prácticamente ignoraron para la enseñanzà secundaria y universitaria, si bien los consideraron importantes en las primeras etapas de la escolarización (Kerr, 1963).

En los primeros años de la década de los setenta, ya se señala que para mejorar la eficacia del trabajo práctico hay que superar el obstáculo que suponen las diferentes expectativas que sobre él tienen los colectivos de profesores y estudiantes; para ello se propone una clarificación de los objetivos por parte de los profesores y su comunicación efectiva a los estudiantes, especificando el tipo de experiencias a realizar y sus propósitos educativos, así como la necesidad de estrechar los vínculos entre lo que se realiza en el laboratorio y el contenido de las clases teóricas.

Hofstein y Lunetta (1982) denuncian que muchos de los objetivos de las prácticas que se plantean en los informes de los años setenta, son los mismos que los objetivos generales de un curso de ciencias, por lo que señalan la necesidad de definir concretamente dónde el trabajo de laboratorio puede aportar algo especial, propio y significativo, para poder explotar adecuadamente esta particular forma de enseñanza. Como ejemplo de formulàción de esta clase de objetivos inespecíficos citan trabajos como el de Anderson (1976), en el que se 
proponen cuatro aspectos educativos a desarrollar mediante el trabajo práctico:

1. EI laboratorio es el lugar donde una persona o un grupo emprende la tarea humana de examinar e intentar proporcionar una explicación a los fenómenos naturales.

2 El trabajo de laboratorio da la oportunidad de aprender formas de razonamiento sistemáticas y generalizadas que pueden ser transferidas a otras situaciones problemáticas.

3. El laboratorio permite al estudiante apreciar, y en parte cmular, el papel del científico en Ia investigación.

4. El trabajo de laboratorio proporciona una visión de conjunto de las distintas ciencias, que incluye no sólo las líneas maestras de sus interpretaciones sobre la naturaleza, sino también la naturaleza provisional y tentativa de sus teorías y modelos.

Una revisión reciente sobre el uso del laboratorio en la instrucción en ciencias propone también cuatro objetivos que los autores definen como los característicos y únicos que deben cumplir las prácticas (Lazarowitz y Tamir, 1994), aunque, en nuestra opinión, dichos objetivos no son ni específicos ni exclusivos de ellas:

1. Proporcionar experiencias concretas y oportunidades para afrontar los errores conceptuales de los estudiantes.

2. Dar la oportunidad de manipular datos por medio de los ordenadores.

\section{Desarrollar destrezas de razonamiento lógico y de organización.}

\section{Construir y comunicar valores relativos a la naturale- za de las ciencias.}

Otros investigadores han propuesto la necesidad de considerar diversos enfoques del concepto de trabajo práctico, en un intento de análisis de un asunto que a todas luces es demasiado complejo para poderlo abarcar de forma homogénea con un único término (Hodson, 1988). Así, Boud y otros, 1986) proponen dos enfoques distintos para el trabajo práctico:

1. Un enfoque disciplinar, en el que las actividades prácticas se relacionan con las ideas clave de las disciplinas, ya sean éstas de carácter teórico o práctico. Consideran importante este enfoque en la formación de futuros científicos con la responsabilidad académica de contribuir con su trabajo al avance de las ciencias.

2. Un enfoque sobre las necesidades profesionales, que se considerá necesario en aquellas carreras que tienen un fuertc componente profesional de carácter práctico, como pueden ser medicina o agronomía. Aquí, el trabajo práctico se ciñe a problemas comunes que se presentan en el ejercicio profesional, y que no tienen que estar necesariamente vinculados con los principios científicos en que se basan.
Para Woolnough y Allsop (1985), el trabajo práctico posee tres objetivos fundamentales, y para cl cumplimiento de cada uno de ellos proponen una clase distinta de trabajo práctico:

1. Ejercicios, diseñados para desarrollar técnicas y destrezas prácticas.

2. Investigaciones, en las que los estudiantes tienen la oportunidad de enfrentarse a tareas abiertas y ejercitarse como científicos que resuelven problemas.

3. Experiencias, en las que se propone que los alumnos tomen consciencia de determinados fenómenos naturales.

Naturalmente, esta pareja de investigadores admite la existencia de diseños prácticos híbridos que sean capaces de cumplir varios de estos tres tipos de objetivos simultáneamente, y no consideran en ningún caso que el objetivo del trabajo práctico que realizan los estudiantes sea reforzar y comprobar la teoría correspondiente. Para este tipo de objetivo opinan que es mucho más efectiva la demostración por parte del profesor, ya que la reconstrucción de la teoría a partir del trabajo práctico de los estudiantes no sólo se ha mostrado totalmente inoperante en su objetivo de mejorar su entendimiento de los aspectos teóricos de las disciplinas (Gunstone, 1991; Watson et al., 1995; Woolnough, 1991), sino que incluso puede ir en detrimento de la calidad del mismo trabajo práctico y del nivel de comprensión de los fundamentos teóricos por parte de los estudiantes (Woolnough y Allsop, 1985).

Las reformas curriculares que se realizaron en los años ochenta, muchas de ellas aún en vigor, pusicron un énfasis mayor en los procesos y procedimientos de las ciencias en detrimento de la enseñanza de las ciencias como cuerpos de conocimiento (Hodson, 1992a; Wellington, 1989); este movimiento ha afectado fuertemente a las prácticas de laboratorio, que se han venido centrando cada vez más sobre los procesos, llegando algunos responsables de los diseños curriculares no sólo a identificar el conocimiento procedimental como un aspecto separado de la actividad científica, sino incluso como el aspecto más importante y, por tanto, el fundamental para la enseñanza de las ciencias (Millar, 1991). Esto conlleva que los currículos de estas características propongan para las prácticas de laboratorio objetivos como los descritos en los proyectos curriculares de Inglaterra y Gales (DES, 1985):

\section{Hacer observaciones.}

2. Seleccionar entre las observaciones realizadas las relevantes para las investigaciones que se plantea realizar.

3. Buscar e identificar regularidades y patrones, relacionándolos con otros ya percibidos anteriormente.

4. Sugerir y evaluar explicaciones para los patrones propuestos. 
5. Diseñar y realizar experimentos para probar las explicaciones sugeridas para los patrones de regularidad propuestos.

Fste enfoque educativo que enfatiza la enseñanza descontextualizada de los procesos científicos, en el que el trabajo práctico se considera como un vehículo para enseñar y desarrollar destrezas cognitivas transferibles y de alto nivel, es absolutamente insostenible. No se pueden enseñar los procesos cognitivos generales como observar, clasificar, realizar hipótesis, etc. per se, como si fueran abstractos; son procesos no generalizables y no transferibles, fuertemente dependientes de la teoría correspondiente y estrechamente ligados a ella (Hodson, 1992a; Millar, 199 I). Los niños ya saben observar, clasificar y hacer hipótesis por ellos mismos, son procesos que realizan continuamente en su quehacer diario. Lo que nosotros queremos enseñarles realmente es la observación científica, la clasificación científica y la formulación científica de hipótesis; por ejemplo, la clasificación biológica no es un asunto trivial de similaridades y diferencias, sino que hay que aprender a reconocer las categorías taxonómicas producidas por la teoría sistemática.

Además, la ciencia escolar se enseña como si la naturaleza de la práctica científica fuese inductiva. Esta imagen deriva de la concepción de método científico formulada por Bacon, en la que se considera que es un proceso con una serie de pasos consecutivos y característicos (Cawthron y Rowell, 1978):

\section{Observación y experimentación}

2. Generalización inductiva

3. Formulación de hipótesis

4. Intento de verificación

\section{Comprobación o rechazo}

\section{Obtención de conocimiento objetivo}

Esta concepción de los científicos como empiristasinductivistas es muy común entre los estudiantes y, desgraciadamente, entre numerosos profesores (Désautels et al., 1993; Gil, 1993; Meichstry, 1993; Praia y Cachapuz, 1994; Smolicz y Nunan, 1975), a pesar del poco apoyo que recibe en la actualidad del resto de colectivos que tienen que ver con las ciencias. Hoy se considera la observación dependiente de la teoría; es la teoría la que determina qué y cómo hay que observar.

Esta visión fuertemente inductivista del método científico, que lo contempla como una sucesión de pasos discretos a los que denomina procesos, ha recibido numerosas y contundentes críticas, y en la actualidad está desacreditada en numerosos sectores, pero dista mucho de haber sido erradicada del mundo de la ensenanza de las ciencias (Millar y Driver, 1987).

A la vista de la gran disparidad de opiniones sobre los objetivos que se persiguen con el trabajo práctico en la enseñanza de las ciencias, parece sensato concluir que no ha sido satisfecha la necesidad urgente de consenso y clarificación de dichos objetivos en el colectivo de profesores, investigadores y responsables del desarrollo curricular. En la concepcion actual del aprendizaje de las ciencias, que sintetiza principios de la psicología educativa y de la filosofía y epistemología de las ciencias, los objetivos del trabajo práctico continúan sin ser precisados. Así, cuando se nos pregunta hoy a los profesores sobre la base que fundamenta nuestra creencia en que el trabajo práctico es una herramienta imprescindible para la enseñanza de las ciencias, seguimos proporcionando una gran variedad de respuestas, muchas de ellas simplistas e idénticas a las que se tenían por evidentes en el marco de la enseñanza por descubrimiento (Lynch, 1987; Miguens y Garret, 1991; Tobin, 1986), y otras que carecen de una relación directa con la enseñanza de las ciencias, por ejemplo, promover el trabajo cooperativo y la discusión entre iguales (Head, 1982).

Hodson (1992a y 1994) clasifica en seis categorías las respuestas específicamente relacionadas con la ensenanza de las ciencias que proporcionamos los profesores acerca de los objetivos de los trabajos prácticos:

1. Para motivar, ya que estimulan el interés y son entretenidas.

2. Para desarrollar actitudes científicas.

3. Para mejorar el aprendizaje del conocimiento científico.

4. Para adiestrarse en el método científico.

5. Para enseñar las técnicas de laboratorio.

6. Para desarrollar la capacidad de llevar a cabo investigaciones cientificas y obtener experiencia de ello.

Sin embargo, la mayoría de estos objetivos han sido cuestionados por los resultados obtenidos en muchas de las investigaciones que se han realizado sobre el trabajo práctico en los últimos años. De hecho se puede decir que casi todos los trabajos publicados desde los años ochenta hasta hoy sobre este tema se dedican especialmente a señalar los aspectos negativos del trabajo práctico que se realiza en la enseñanza de las ciencias, tanto desde el punto de vista de los objetivos planteados como desde el grado de su consecución, si bien todos ellos mantienen en común la firme convicción de que el trabajo práctico es imprescindible para un adecuado proceso de enseñanza y aprendizaje de las disciplinas científicas.

Los estudios acerca de la percepción que tienen los alumnos de la importancia de las prácticas muestran en general que ellos no sostienen los mismos puntos de vista que los investigadores y profesores (Boud et al., 1980; Friedler y Tamir, 1986; Gardner y Gauld, 1990; Kirschner et al., 1993). Por ejemplo, respecto de la motivación que se supone que les proporciona para seguir estudiando ciencias, un estudio sobre el trabajo de Iaboratorio realizado en bioquímica revela que sólo un $50 \%$ de los estudiantes muestra algún grado de entusiasmo por él, lamentándose además de la pobre recompensá 
intelectual que reciben a cambio de tantas horas de dedicación en la bancada (Tremlett, 1972). En una investigación etnográfica llevada a cabo en dos institutos australianos, sólo treinta de ochenta y seis alumnos consideraron que el trabajo de laboratorio era la actividad de las clases de ciencias que más les gustaba; además, únicamente quince de ellos declararon que habian aprendido más sobre las ciencias participando en actividades prácticas; $y$ un total de veinte depositaron su confianza en que el currículo de ciencias mejoraría si se aumentaba el tiempo dedicado al trabajo científico. No obstante, el autor del trabajo concluye que sus datos demuestran que el trabajo práctico es una actividad motivadora para los estudiantes de ciencias (Tobin, 1986).

Por otra parte, si bien algunos estudios parecen demostrar que la mayoría de alumnos disfrutan con las prácticas de laboratorio (Friedler y Tamir, I990; Lazarovitz y Tamir, 1994), también hay otros que muestran que el interés en ellas decrece fuertemente con la edad (Lynch y Ndyetabura, 1984), e incluso que existe una minoría significativa que expresa aversión por el trabajo práctico (Bliss, 1990; Head, 1982).

En cuanto a la capacidad de las prácticas para desarrollar actitudes científicas, en primer lugar habría que preguntarse cuáles son las características propias y distintivas de Ios científicos $y$, en caso de encontrarlas, si son deseables en sí mismas como objetivo educativo general. Hodson (1993 y 1994) mantiene que estas actitudes científicas no son en realidad más que las que se atribuyen a la imagen estereotipada del científico: un varón maduro, imparcial, dispuesto siempre a considerar las ideas de los demás y a compartir las propias, que evita la emisión de juicios apresurados y cuya objetividad está exenta de prejuicios. Además de dudar de la existencia real en el colectivo científico de tal idiosincrasia, Hodson opina que sería mucho más positivo en el plano educativo presentar al científico como una persona normal, afectuosa, divertida, diligente, etc., y aun sería más interesante proporcionar la idea de que cualquier persona con cualidades normales puede convertirse en científico.

Las otras cuatro categorías de respuestas propuestas por Hodson (1993 y 1994) están directamente relacionadas con el proceso de aprendizaje de las ciencias y asumen la idea de que el trabajo práctico debe proporcionar aprendizaje significativo. Sin embargo, parece que los alumnos no consideran el trabajo práctico como una estrategia de enseñanza especialmente valiosa para el aprendizaje. En el estudio ya comentado del sindicato de estudiantes de Inglaterra, se incluye un análisis de las opiniones de los estudiantes de ciencias de cinco de sus universidades, entre las que destaca su consideración del trabajo práctico como el medio menos valioso de todos los empleados para la enseñanza, flegando a declarar un $17 \%$ de Ios estudiantes que las prácticas son absolutamente ineficientes en la enseñanza recibida.

Esta cuestion fue ya discutida hace tiempo por Novak (1978) en el contexto del aprendizaje por descubrimien- to, concluyendo que el trabajo práctico no tiene necesariamente que conducir al aprendizaje significativo; no obstante, esta conclusión fue minusvalorada entonces al ser entendida como una condena al trabajo de laboratorio en favor de una pedagogía de transmisión oral, cuando quizás sus intenciones no iban más allá áe aconsejar que había que reexaminar la calidad y los objetivos del trabajo práctico que se realizaba en la enseñanza antes de realizar juicios sobre su validez.

Parece que recientemente se ha empezado a realizar este «reexamen», y uno de los autores que aborda esta tarea es Kirschner (1992). En su trabajo discute que el error fundamental de los curriculos centrados en los procesos de las ciencias es que presuponen que aprender ciencias puede ser equivalente al proceso de investigación científica, una idea que como mínimo es opinable, y que para Kirschner representa un grave error conceptual causante de efectos muy perjudiciales en la enseñanza de las ciencias. Él parte de que se comete un error básico de tipo epistemológico cuando se plantean los objetivos del trabajo práctico en la enseñanza de las ciencias: se asume que el contenido pedagógico de la experiencia de aprendizaje es idéntico a la estructura sintáctica de la disciplina que se estudia; es decir, se confunden Ias bases psicológicas y las epistemológicas de enseñar ciencias, no se sabe distinguir entre hacerciencia, aprender ciencias y aprender sobre las ciencias (Hodson, 1992b). Se presupone que la práctica de las ciencias es la mejor forma de enseñarlas, y por tanto de aprenderlas, pero la realidad muestra que es demasiado simplista astumir que una teoría de educación puede destilarse directamente a partir de una filosofía de las ciencias.

Existen grandes diferencias entre el científico y el aprendiz de ciencias; Anderson (1976) define muy bien la relación entre el científico, el acto de hacer ciencia y las mismas ciencias:

- El científico es una persona con un papel social dedicado a la investigación y a la acumulación de conocimiento, que posec no sólo un vasto dominio de un campo de conocimiento, sino también un desarrollado sentido de la predicción sobre los fenómenos naturales

- Hacer ciencia es la aplicación de los métodos de investigación, tanto físicos como intelectuales, para estudiar las propiedades enigmáticas de la naturaleza.

- Las ciencias son cuerpos de conocimiento y metodología que se han formado por acumulación gracias a la utilización continuada por parte de los científicos de los procesos de hacer ciencias; son también una manera de razonar y sistemas de metodologías de que se dispone para realizar la tarea de interpretar los fenómenos naturales.

Los estudiantes no poseen ni el suficiente dominio de un cuerpo de conocimiento, ni la sofisticación teórica, ni la amplia experiencia de un científico (Norris, 1995), con lo cual todos los currículos que se plantean que el alumno aprenda ciencias a partir de su propia experimentación están condenados al fracaso. Kirschner (1992) 
aún va más lejos al rechazar que el trabajo práctico tenga valor en sí mismo para mejorar el conocimiento de las teorias cientificas en la enseñanza, un objetivo reconocido ampliamente por el colectivo de profesores y disenadores curriculares. Esta crítica la fundamenta en la relación interdependiente e interactiva de que gozan la teoría y la experimentación en el proceso de hacer ciencia. I os experimentos ayudan a construir la teoría y la teoría determina el tipo de experimentos que se realizan. El científico que experimenta es siempre un teórico, ya que de otra forma sería incapaz de interpretar los resultados que obtiene. I os currículos prácticos en la enseñanza se han esforzado durante mucho tiempo en proporcionar experimentos que comprueben los principios científicos, experimentos donde siempre surge un resultado correcto si se siguen adecuadamente las instrucciones. Esta manera de concebir el trabajo práctico no sólo no enseña la estructura sintáctica de la disciplina, sino que refuerza la visión empirista de los estudiantes y presenta las ciencias como cuerpos de conocimientos verdaderos e inmutables. Además, los estudiantes no se motivan por este tipo de prácticas de laboratorio, que les producen apatía y aburrimiento hacia las ciencias y el trabajo científico.

Kirschner concluye que, si bien las prácticas no son particularmente útiles para proporcionar a los estudiantes conocimiento sobre la estructura sustantiva de las disciplinas científicas, sí pueden ser utilizadas para introducirlos en la estructura sintáctica đel conocimiento científico. Para ello propone tres motivos para realizar trabajo práctico en el laboratorio, que él defíne como válidos y novedosos y, en correspondencia con cada uno de ellos, asigna un tipo concreto de práctica de los previamente definidos por Woolnough y Allsop (1985):

1. Desarrollar destrezas especificas, para lo que se proponen los ejercicios, y en los que las simulaciones educativas pueden dar mu buen rendimiento.

2. Ensefar el enfoque académico del trabajo científico, para lo que propone la realización de investigaciones, incluyendo algunas de las muchas tareas que realiza un científico cuando restuelve problemas:

- Fstudiar una situación y apercibirse de que existe un problema por resolver.

- Definir claramente el problema a resolver.

- Buscar estrategias đe resolución para el problema.

- Evaluar las estrategias alternativas planteadas.

- Iispecificar o elegir la estrategia que considera más valiosa.

- Resolver el probiema.

- Evaluar la solución y estudiarla para determinar si con ella aparece un nuevo problema.

3. Permitir que los estudiantes tomen experiencia de los fenómenos, mejorando su conocimiento tácito, para lo cual propone las experiencias. No se trata aquí de adquirir conocimientos científicos de los fenómenos por me- dio del trabajo práctico, sino de obtener consciencia implícita -que en la mayoría de las ocasiones no se puede verbalizar-sobre to que ocurre con un fenómeno, no sobre cómo o por qué ocurre.

Así, a pesar de la investigación abundante realizada sobre los trabajos prácticos, los resultados obtenidos no despejan las principales incógnitas planteadas. Posiblemente esto se deba, por una parte, a la falta de coherencia y uniformidad que presentan entre sí los diversos fenómenos y estrategias que solemos agrupar bajo los términos ambiguos de trabajos prácticos o prácticas de laboratorio y, por otra, a los supuestos implícitos que subyacen en muchas de estas investigaciones y que las orientan en direcciones determinadas, impidiendo así apreciar otras posibles interpretaciones o soluciones a las cuestiones planteadas.

En lo que sí parecen estar de acuerdo tanto profesores como estudiantes es en que el auténtico valor de las prácticas reside en el desarrollo de actitudes y destrezas cognitivas de alto nivel intelectual, más que en la adquisición de destreza manual y de técnicas de manipulación; sin embargo, también están de acuerdo en que hay muy pocos indicios de que el trabajo práctico que realmente se realiza en la enseñanza ayude a la consecución de estos objetivos.

A la vista de las investigaciones que se han venido realizando en los últimos años sobre los objetivos asig. nados al trabajo práctico en la enseñanza de las ciencias, podemos concluir que existe una marcada tendencia a plantear objetivos específicamente ligados a la idiosincrasia experimental de las ciencias, abogando por el abandono de otros fuertemente arraigados, como la motivación, la comprobación de la teoría o el desarrollo de destrezas cognitivas de nivel alto, que se han mostrado inabordables a través del trabajo de laboratorio de los estudiantes. En algunos de los trabajos publicados que intentan fundamentar una propuesta alternativa, se constata la necesidad de producir tipos específicos de prácticas para cumplir cada uno de los objetivos planteados, quizás en un intento de clarificar algunos aspectos que se han mostrado extraordinariamente complejos en su anitIisis. Eso sí, todos los informes finalizan reconociendo la necesidad del trabajo práctico en la enseñanza de las ciencias y reciamando una mayor dedicación a la investigación de este tema crucial en la educación científica.

\section{¿QUÉ SE OBTIENE DEL TRABAJO PRACTICO?}

Si la situación del trabajo práctico en cuanto a la formulación de sus objetivos en un currículo de ciencias es muy diversa y no ha conseguido un consenso, la que mantiene respecto del cumplimiento de dichos objetivos tampoco es la más adecuada. Algunos investigadores han llegado a considerar que el trabajo práctico de laboratorio es una pérdida de tiempo y recursos (Hofstein y Lunetta, 1982; Pickering, 1980; Toothacker, 1983). Otros muchos han demostrado que los objetivos 
que se esperan cubrir con este tipo de enseñanza no se cumplen (Igelsrud y Leonard, 1988; Lederman, 1992; Reif y St. John, 1979; Tamir y Lunetta, I978 y 1981 ; Tobin, 1986; White, 1979). La mayoría concluyen que el trabajo práctico que realmente se realiza en la enseñanza actual de las ciencias son experiencias tipo receta para aprender sobre las ciencias, para confirmar hechos y teorías mediante la obtención de los resultados correctos, en lugar de realizar investigaciones más amplias de la naturaleza por medio de la exploración, la investigación, la comprobación y la explicación (Kyle et al., 1979; Qualter et al., 1990; Tamir, 1977; Tamir y García, 1992; Tobin, 1986; Tobin y Gallagher, 1987). Los estudios que han comparado la eficiencia del aprendizaje siguiendo un método de trabajo práctico con otros que han seguido metodologías más convencionales de enseñanza no han obtenido resultados positivos, queriendo decir con esto que el laboratorio se ha mostrado, en el mejor de los casos, igual de eficiente que los métodos convencionales de instrucción a la hora de mejorar las variables de aprendizaje medidas en los alumnos (Hofstein y Lunetta, 1982; Clackson y Wright, 1992). Incluso se ha constatado en alguna ocasión que puede liegar a ser menos útil en lo que concierne al aprendizaje de conceptos que otras estrategias como, por ejemplo, las denostadas demostraciones llevadas a cabo por los profesores (Thijs y Bosch, 1995). También es cierto que los resultados de esta clase de trabajos, que se basan en una metodología de investigación que intenta contrastar la eficiencia de un método de ensef̃anza $x$ con la de otro $y$, han sido puestos en duda, ya que se parte de la presunción de que existe un método único para el trabajo de laboratorio planteado a los estudiantes y otro para las demostraciones de los profesores, olvidando la enorme diversidad de enfoques que ambos pueden adoptar, y dejando de lado una gran cantidad de variables que sin duda afectan fuertemente a los resultados obtenidos (Atkinson, 1990; Garret y Roberts, 1982; Shulman y Tamir, 1973).

Pero a pesar de todo, este tipo de conclusiones produce la incómoda sensación de que el trabajo de laboratorio proporciona muy poco beneficio a los estudiantes en su tarea de comprensión de los conceptos científicos. Parece que Ia única justificación para el trabajo práctico que se realiza es el moderado éxito que produce en Ia enseñanza de técnicas de medida y en la mejora de la destreza manual de los alumnos (Bryce y Robertson, 1985; Hegarty-Hazel, 1990a; Stawinski, 1986), habilidades que sería más apropiado adquirirlas por medio de otro tipo de actividades manuales, y de las que se duda que proporcionen algún valor educativo por sí mismas, al menos en la enseñanza no universitaria (Hodson, 1990).

Algunos investigadores han estudiado los efectos a largo playo que produce el trabajo de laboratorio en los estudiantes, y los resultados tampoco son alentadores. Un informe realizado sobre estudiantes de la licenciatura de física puso de manifiesto que ellos no recordaban en absoluto más de la mitad de las experiencias de física que habían realizado en el laboratorio durante su etapa de educación secundaria, lo cual plantea la pregunta de que si ésta es la eficiencia que consigue el trabajo práctico en los alumnos capacitados y motivados que han seguido estudiando física, ¿qué valor tendrá este método educativo para todos los demás? (Clakson y Wright, 1992).

Tampoco parece que un sistema educativo con una larga tradición en el uso del trabajo práctico y con una gran dedicación a él en la instrucción sea capaz de producir beneficios académicos tangibles a sus estudiantes de ciencias. Un estudio comparativo sobre el diferente comportamiento de alumnos de educación secundaria de Inglaterra y España en el Jaboratorio de química que estudian el fenómeno de la combustión (Watson et al., 1995) concluye que, si bien los ingleses han tenido mucha más experiencia directa en el laboratorio sobre esta reacción química, esta experiencia no ha sido capaz de modificar sus esquemas mentales alternativos sobre la combustión. La comprensión que obtienen de este fenómeno es absolutamente similar a la que muestran los estudiantes españoles, a pesar de que la experiencia práctica de estos últimos es mucho más limitada y que las respuestas que proporcionan en los cuestionarios utilizados en la investigacion son cualitativamente diferentes debido a sus distintas experiencias educativas. Todos ellos, ingleses y españoles, mantienen sus ideas erróneas sobre la combustión en un esquema mental coherente con el propuesto por Driver (1985) como prototipo de combustión. Este resultado parece indicar que el efecto de un currículo como el inglés, en al que un $60 \%$ del tiempo de enseñanza de química en la educación secundaria es ocupado por trabajo práctico, no produce en sus estudiantes un mayor rendimiento de cambio conceptual que el que es constatado en Ios que siguen el currículo español, con una dedicación al trabajo práctico bastante inferior a la mitad y siendo además la mayor parte de ella empleada en demostraciones realizadas por el profesor, algo poco habitual en las escuelas inglesas (Watson y Pricto, 1994).

Tanto alumnos como profesores somos conscientes del escaso rendimiento que se obtiene del trabajo práctico -y no hay que olvidar que desafortunadamente se puede decir lo mismo del trabajo «teórico». Ya en los años sesenta se propuso, en una serie de informes sobre la educación científica en Australia, Estados Unidos e Inglaterra, la necesidad de reducir drásticamente el tiempo dedicado al laboratorio de ciencias en la enseñanza, eliminando todos los experimentos de tipo receta, y de diseñar trabajos experimentales de mayor valor educativo -sin especificar claramente a qué se referían con este calificativo. También entonces, alumnos de universidades inglesas criticaban el número de horas excesivo que se dedica al trabajo práctico ( 10 horas semanales de media) y estimaban conveniente una reducción (Tremlett, 1972).

En la actualidad, la situación es similar, quizá porque no se ha encontrado remedio en los últimos veinte años. Ésta es la razón principal por la que, a pesar de seguir siendo reconocido el trabajo práctico como un componente crítico en la instrucción científica, se haya ido paulatinamente perdiendo la confianza en que el laboratorio es un lugar efectivo para aprender ciencias (NRC, 1990; Stake y Easley, 1978). Un informe reciente sobre 
el estado del trabajo práctico en la enseñanza de la biología en setenta y tres universidades estadounidenses es un claro reflejo de esta pérdida de confianza (Sundberg y Armstrong, 1993). En el informe se observa una tendencia en la mayoría de las universidades de este país, tanto públicas como privadas, a reducir alguno de los componentes del trabajo práctico considerados críticos por los autores: personal, espacio (físico y temporai), financiación y apoyo. Las explicaciones proporcionadas para justificar el recorte son variadas, entre ellas: 1) el aumento de alumnos matriculados hace difícil el proporcionarles un trabajo práctico adecuado, con lo que Ia opción elegida es climinarlo, al menos parcialmente; 2) la reducción de ingresos, el aumento de gastos y el consiguiente recorte económico repercute especialmente en el presupuesto dedicado al trabajo práctico; 3) la eliminación del trabajo práctico en el primer curso de carrera se justifica por considerar que el trabajo práctico que allí sé plantea ya ha sido realizado por los alumnos en su instrucción anterior, y así se puede disponer de más fondos para manejar equipos de laboratorio mejores en los últimos cursos de la carrera; 4) el reconocimiento de que la mayoría del trabajo práctico realizado no produce un rendimiento adecuado y la ausencia de alternativas válidas conduce a la decisión de reducir el tiempo dedicado a la enseñanza en el laboratorio. Se pueden detectar tendencias similares en diversas universidades europeas, especialmente en el período actual de reforma profunda que están experimentando la mayoría de ellas (Science, 1996).

En los últimos años, numerosas investigaciones han intentado buscar una justificación a estos malos resultados, y una de las conclusiones que comparten muchas de ellas es que muchos de los trabajos realizados para medir la eficiencia de las prácticas en la instrucción científica han utilizado instrumentos de medida inadecuados, han sido deficientemente planteados o poseen fuertes limitaciones para considerar generalizables sus resultados. En un artículo reciente se puede leer:

Hay muchas limitaciones de este estudio que precisan ser tenidas presentes antes de interpretar los resultados. Primero, este estudio sólo investiga las actitudes de los estudiantes sobre las experiencias de laboratorio en biología, por lo que no pueden ser generalizadas a otras ciencias como física, quimica o ciencias de la Tierra, ni tampoco a otras formas de instrucción utilizadas en el curriculo de biología. Además, ya que aquí se han estudiado los alumnos de los grados 10,11 y 12, los resultados no deben generalizarse a otros niveles educativos. Por último, este estudio se ha llevado a cabo en una escuela privada, preparatoria para la universidad, con un grado alto de opcionalidad por parte de sus alumnos sobre la elección de sus materias de ciencias. Esta característica, así como otras que son únicas en este ambiente escolar, puede limitar la generabilidad de los resultados (Weinburgh y Englehard, 1994).

Esta declaración ejemplar por parte de los autores sobre el rango de aplicación de los resultados obtenidos es, desgraciadamente, muy poco frecuente en los informes de investigación. Muchos de los trabajos publicados sobre este tema carecen de una descripción detallada del tipo de práctica de laboratorio realizada, de las variables que pueden afectar al instrumento de medida utilizado, de los objetivos planteados tanto para la instrucción práctica como los de la propia investigación, etc., con Io que la lectura de sus resultados producc una idea vaga y general, frecuentemente negativa, de la eficiencia del trabajo práctico en la enseñanza de las ciencias, idea que por otra parte se ajusta bastante a ese sentimiento general compartido por muchos profesores y estudiantes acerca de la falta de eficacia de las prácticas. Pero este tipo de trabajos aporta poco a una posible solución de los problemas intuidos, ya que ni siquiera permite su formulación adecuada.

Una cuestión de difícil respuesta es si la forma de evaluar las prácticas es la responsable de los resultados obtenidos; es decir, si los instrumentos de medida utilizados son adecuados a los propósitos para los que han sido diseñados. Así, en un trabajo sobre las prácticas de biología en la educación secundaria, se concluye que los instrumentos de medida utilizados en la investigación deben corresponderse estrechamente con los objetivos específicos de la educación experimental en biología (Stawinski, 1986). Sus resultados muestran que, si únicamente se utilizan pruebas de conocimientos en la investigacion, podemos obtener la conclusión errónea de que la instrucción experimental en biología produce sólo un débil efecto positivo y no significativo en el aprendizaje. La mayoría de investigaciones están de acuerdo en señalar que los métodos clásicos de evaluación utilizados, como las pruebas de lápiz y papel, no son adecuados para medir situaciones de resolución de problemas o de adquisición de destrezas (Doran et al., 1994; Lazarowitz y Tamir, 1994; Tamir, 1974).

Lo que sí parece claro es la fuerte influencia que ejerce la forma de evaluación del trabajo práctico sobre su propio (Jenkins, 1995a y 1995b). Esto parece que no sólo ocurre con el trabajo práctico, sino que es un problema común a toda la enseñanza de las ciencias; pero en el caso del trabajo práctico parece que la situación es aún más grave (Woolnough y Toh, 1990). Cuando un sistema educativo posee evaluaciones externas a gran escaia, como exámenes nacionaies para acceder a la universidad o a la educación secundaria, la docencia que se realiza está fuertemente condicionada por la evaluación externa (Black, 1995). En los casos en que el trabajo práctico no es evaluado en esta clase de pruebas sumativas de amplia distribución, se ha constatado que ha disminuido notablemente su uso como herramienta para Ia enseñanza de las ciencias, Ilegando incluso a correr el riesgo de desaparecer del currículo (Black (1993). Sin embargo, cuando ha sido introducido como objeto a evaluar por parte de estas pruebas, se suele concentrar la atención en la evaluación de la capacidad de cada individuo para realizar correctamente un conjunto de tareas determinadas a priori como objetivos educativos. Naturalmente, este enfoque sobre la medida personal de destrezas deja de tado las funciones formativas de la evaluación, tanto si el tipo de exámenes es externo como si es interno, ya que relega al evaluador a un papeI de técnico que aplica una guía detallada de objetivos prede- 
terminados de la estrategia evaluadora planeada por supuestos especialistas educativos.

Este enfoque que enfatiza las destrezas prácticas en el trabajo de laboratorio es el mayoritario en los países anglosajones (Bryce y Robertson, 1985; Buchan,1992; Doran et al., 1994), así como sus correspondientes formas de evaluación referidas a criterios sobre las destrezas prácticas adquiridas (proyectos APU o TAPS: Johnson, 1989; Bryce et al., 1983, respectivamente), y es heredero de las tendencias reduccionistas de los años setenta en la enseñanza de las ciencias (Woolnough, 1991). Estas tendencias, que nacieron con la sana intención de clarificar los objetivos educativos siguiendo el camino iniciado por la taxonomía de Bloom (1956), definieron conjuntos de objetivos de comportamiento mensurables. Con la obsesión de medir lo más exactamente posible y de que las medidas fuesen frables, los objetivos educativos han ido desplazándose hacia aquéllos que son más mensurables, obviando los que no lo son; la actividad científica en las aulas se ha dividido en un conjunto de partes fácilmente mensurables que, además de no ser las más importantes, olvidan la parte holista de las ciencias al asurnir que la suma de las partes proporciona el global. La obsesión de este enfoque sobre las destrezas por asegurar su fiabilidad y eliminar la ambigüedad de las medidas descansa sobre una idea de las ciencias absolutamente errónea. Hacer ciencia es un proceso difuso, incierto, intuitivo e idiosincrásico, y debe apreciarse en la enseñanza con toda su vaguedad, sin intentar disimularla. En este contexto, la noción de que todos los estudiantes deben alcanzar cierta puntuación en cada uno de los atributos de una lista de objetivos previamente prescrita no sólo es una práctica eđucativamente poco aconsejable en la enseñanza de las ciencias, sino que además carece de sentido. De nuevo insistimos en que hay que guardarse de infravalorar aquello que no somos capaces de medir con exactitud y en que no hay que olvidar que, si bien no Io podemos medir, siempre seremos capaces de emitir un juicio sobre ello.

\section{¿QUÉ SE PUEDE HACER CON LAS PRACTICAS?}

Como hemos podido ver, la investigación que se ha llevado a cabo hasta ahora en general no se ha mostrado capaz de proporcionar un soporte empírico sólido para el papel del trabajo práctico en la enseñanza de las ciencias. Esta falta de definición en sus resultados sobre los objetivos y la efectividad del trabajo práctico es sin duda debida en parte a la complejidad del objeto de investigación, pero también a que muchas de las investigaciones realizadas están deficientemente estructuradas y sus resultados carecen de fiabilidad (NRC,1990). Muchas de ellas han fallado en su intento al adoptar un enfoque poco preciso sobre el tipo de trabajo práctico que se estaba investigando y sobre los objetivos educativos concretos para los que había sido diseñada. Es necesario considerar los distintos tipos de prácticas que se realizan, los objetivos que se persiguen, el currículo en el que se integran, etc., y planear cuidadosamente la investigación siendo consciente de ello (Duggan y Gott, 1995). La principal diferencia entre los tipos de trabajo práctico que se realizan en la instrucción se establece entre el que se planea para mejorar el conocimiento de los alumnos sobre las teorías científicas y el que está destinado a desarrollar las destrezas cognitivas necesarias para resolver problemas científicos. Ambas aproximaciones han sido teóricamente desacreditadas por numerosos investigadores, siendo a menudo calificadas de reduccionistas, y los resultados de investigación que se han obtenido sobre su eficiencia son poco concluyentes y normalmente desalentadores.

Además del tipo de prácticas que se realiza, también es preciso tener en cuenta el nivel educativo concreto para el que han sido diseñadas a la hora de plantear una investigación sobre su efectividad. En general, se ha prestado muy poca atención al trabajo práctico que se realiza en la escuela primaria, a pesar del reconocimiento unánime de que este enfoque de manos a la obra es absolutamente imprescindible para un aprendizaje significativo en estos niveles educativos. En la enseñanza secundaria quizás habría que distinguir entre el trabajo práctico que se realiza en currículos cuyo principal objetivo es preparar a los alumnos para ser futuros científicos, y el que se hace en aquellos currículos que pretenden proporcionar una alfabetización científica de los estudiantes, conscientes de que muchos de ellos no seguirán estudiando ciencias. No sólo el nivel intelectual, afectivo y de desarrollo de los estudiantes es diferente en los diversos niveles educativos, sino que los mismos objetivos curriculares pueden ser muy distintos según el enfoque adoptado - por ejemplo, ciencia integrada o ciencia disciplinar, o ciencia para todos y ciencia elitista, según la terminología de Fensham (1990)-, lo que sin duda debe tenerse en cuenta a la hora de evaluar la efectividad del trabajo práctico que en ellos se realiza (Edwards y Power, 1990; Friendler y Tamir, 1990). En la enseñanza universitaria, debería esperarse una concepción distinta đel trabajo práctico, ya que en ella los currículos suelen estar orientados a la formación de científicos con capacidad de investigar; es decir, cabría esperar que el aprender a hacer ciencia, el pináculo de la educación científica, fuese el objetivo primordial a conseguir mediante el trabajo de laboratorio -sin olvidar la importancia que esta estrategia educativa puede ofrecer también a la hora de aprender ciencias y aprender sobre las ciencias (Hodson, 1988). No obstante, parece ser que es en este nivel educativo donde resulta más difícil modificar las bien establecidas y tradicionales maneras de enfocar el trabajo práctico, y que las reflexiones y propuestas que se producen sobre los currículos de ciencias del nivel secundario rara vez llegan hasta la universidad, incluso siendo muchas de ellas formuladas precisamente desde esta institución (Hegarty-Hazel, 1990b; Meester y Maskill, 1993, 1994, i995a y 1995b).

Algunos trabajos de investigación, además de no contemplar muchas de estas variables que consideramos fundamentales para su planteamiento, se muestran extraordinariamente ambiciosos en sus resultados y rango 
de aplicación; por ejemplo, un artículo reciente (Fraser et al., 1995) concluye que el instrumento desarrollado y validado, un cuestionario de 35 preguntas, es adecuado para asesorar, evaluar e investigar las clases en el laboratorio, además de para guiar investigaciones sobre los subambientes creados por los subgrupos de trabajo de los alumnos y para la construcción adecuada de estudios de casos individuales. No le restamos valor a este tipo de pruebas de amplia distribución a la hora de proporcionar a los investigadores datos acerca del tipo de trabajo de laboratorio que se realiza en las escuelas, pero sí dudamos de que las respuestas a esas 35 preguntas sean capaces de evaluar eficientemente cualquier tipo de trabajo práctico y de proporcionar a profesores y alumnos un asesoramiento óptimo para su reconceptualización y mejora.

Quizás estemos confundiendo los métodos de investigación que tratan de obtener datos sobre la eficiencia del trabajo práctico en la enseñanza de las ciencias con la evaluación educativa que de él deben realizar los profesores responsables de introducirlo en el curriculo escolar; puede que sea ésta la razón del poco crédito que damos a los resultados negativos de estas investigaciones, ya que seguimos estando todos unánimemente de acuerdo en que el trabajo práctico es vital en la enseñanza de las ciencias. No parece que la actividad práctica de los estudiantes que ha sido diseñada para que contribuya de manera significativa a la comprensión de los conceptos teóricos haya obtenido resultados positivos y, aunque sería interesante realizar mayor cantidad de investigación sobre este punto, quizá sea conventente no supeditar el trabajo práctico a la demostración y comprensión teórica (Kirschner, 1992; Woolnough, 1991 ; Woolnough y Allsop, 1985). Puede que uno de los errores más graves en la educación científica sea el derivado de la concepción de que el trabajo experimental recapitula y comprueba el conocimiento térico, lo que separa en fases sucesivas y estancas la actividad teórica y la experimental, como si esta última fuese un paso definitivo y siempre necesario para comprobar la certeza de las construcciones teóricas previas.

Por otra parte, el análisis del trabajo práctico que se ha planteado como desarrollo de destrezas sobre una taxonomía de objetivos procedimentales y que ha sido evaluado con pruebas referidas a criterios tampoco ha proporcionado datos coherentes y fiables sobre las muchas preguntas formuladas acerca de su efectividad. Este enfoque que presenta el proceso de hacer ciencia de una forma simplista, como si se tratase de una serie lineal de pasos a seguir descritos en un protocolo, y que conlleva las correspondientes herramientas de medida de progreso para su evaluación es totalmente infructuoso.

Queremos insistir de nuevo aquí en que hacer ciencia es una tarea idiosincrásica, no predictible y poco ordenada, que depende de manera crucial del conocimiento tácito del practicante (Toh y Woolnough, 1993; Wolpert, I993). Hodson (1992a) la define como el tipo de conocimiento que posee un ciempiés para permitirle caminar, pero que cuando se le pregunta cómo se las arregla para controlar todas sus patas es incapaz de dar un explicación. Esta visión de lo que es hacer ciencia reclama un enfoque mucho más holista del trabajo práctico en la enseñanza de las ciencias, y debe tener siempre presente que el todo no es igual a la suma de las partes, sino que es mayor y en conjunto más poderoso (Kopfler, 1990; Woolnough, 1991). La capacidad de hacer ciencia es distinta de la de tener ciertas destrezas manipulativas de laboratorio por una parte y un cierto bagaje de conocimiento conceptual por la otra. Si hacer ciencia es una actividad holista, consecuentemente sólo se puede obtener experiencia de ella de manera holista, aprenderla, enseñarla y, por supuesto, evaluarla de forma iguaimente holista.

¿Qué papel puede jugar entonces el trabajo práctico en una enseñanza de las ciencias que sea fiel a sut carácter holista? Quizás sea conveniente reflexionar sobre las características propias y exclusivas que este tipo de actividad puede proporcionar a los estudiantes. Nosotros hemos seleccionado cuatro que pensamos que no sólo caracterizan el trabajo práctico, sino que pueden representar objetivos educativos específicos de él, es decir, que únicamente pueden ser alcanzados por medio de Ia actividad práctica:

1. Proporciona experiencia directa sobre los fenómenos en el sentido de lo que Woolnough y Allsop (1985) denominan experiencias o White (1991) llama episodios, haciendo que los estudiantes aumenten su conocimiento tácito y su confianza acerca de los sucesos y eventos naturales.

2. Permite contrastar la abstracción científica ya esta blecida con la realidad que ésta pretende describir -habitualmente mucho más rica y compleja $\cdots$, enfatizándose así la condición problemática del proceso de construcción de conocimientos y haciendo que afloren algunos de los obstáculos epistemológicos que fue necesario superar en la historia del quehacer científico y que, en cambio, suelen ser omitidos en la exposición escolar del conocimiento científico actual (Astolfi, 1994; Astolfi y Peterfalvi, 1993; Lewis, 1982 y 1988; Watts, 1991).

3. Produce la familiarización de los estudiantes con importantes elementos de carácter tecnológico, desarrollando su competencia técnica.

4. Desarrolla el razonamiento práctico, en el sentido que Brickhouse y otros (1993) definen esta capacidad: es un comportamiento inherentemente social e interpretativo propio de la condición humana y necesario para la praxis, un tipo de actividad en la que el desarrollo progresivo del entendimiento del propósito que se persigue emerge durante el ejercicio de ta propia actividad.

Si diferenciamos to que es aprender ciencias y aprender sobre las ciencias de aprender a hacer ciencia (Abrams y Wandersee, 1995a y b), debemos ser conscientes de que los alumnos sólo aprenderán a hacer ciencia practicándola -al igual que ocurre con los científicos-, siguiendo sus líneas propias de indagación, lo que les permitirá conocer que hacer ciencia no sólo es fuertemente dependiente de la teoría, sino también de la práctica. Todo esto naturalmente no implica que hacer 
ciencia sea un proceso para el que no hay mćtodo, sino sencillamente que no depende de un algoritmo concreto. Los métodos para hacer ciencia naturalmente que existen, pero la naturaleza de cada uno de ellos depende de circunstancias particulares e idiosincrásicas, entre las que el objeto concreto de estudio, el conocimiento teórico previo sobre él, la tradición investigadora y los medios técnicos disponibles son algunas de las más sobresalientes. Que la comunidad científica utilice como criterio de calidad de la investigación los métodos empleados en llevarla a cabo es una muestra clara de la existencia de numerosas y plurales metodologías, así como de su importancia en el proceso científico.

Este enfoque holista del quehacer científico obliga a que la evaluación de su enseñanza, y consecucntemente la del denominado trabajo práctico, se lleve a cabo también de una manera holista, sin intentar aislar y medir el tipo de conocimiento tácito, teórico o las destrezas que se supone que están siendo empleadas. Los profesores deberán utilizar su capacidad de expertos para juzgar la calidad de la investigación realizada por sus alumnos. Una cvaluación adecuada de cómo se hace ciencia debe contemplar la naturaleza personal de la investigación y los cambios de dirección que realizan los estudiantes en cualquicr momento del proceso, así como los motivos empleados para decidir el cambio de dirección (Toh y Woolnough, 1990). Si para poder evaluar imponemos un orden a este proceso, que es de naturaleza interactiva y desordenada, a la vez estamos imponiendo un grado de rigidez que destruye su esencia creativa (Kimbell, $1991)$.

Por tanto, la evaluación holista se presenta no sólo como conveniente, sino necesaria para llevar a cabo en la enseñanza un tipo de trabajo práctico que refleje auténticamente el espíritu del quehacer científico, y naturalmente este tipo de enfoque sólo podrá funcionar con profesores que sean ellos mismos expertos, que tengan experiencia personal en haber realizado investigaciones científicas.

Esta manera de entender la enseñanza de las ciencias y su evaluación es liberadora para los profesores y puede producir en ellos un poderoso estímulo para su desarroIlo profesional. La evaluación referida a criterios del tipo cuestionario de procesos repercute muy negativamente en la clase de trabajo práctico que se realiza, además de suponer para los profesores una carga de trabajo adicional e inútil el intentar comprobar el progreso de todos y cada uno de los puntos detallados en las largas listas de objetivos predeterminados. Una forma de evaluación holista amplía la capacidad del profesor para realizar juicios, y cuando se nos da la responsabilidad y la necesaria formación inicial y permanente, los profesores somos perfectamente capaces de reconocer los aspectos relativos al quehacer científico en los que el estudiante precisa consejo, guía específica, oportunidades para repensar y replantcar su tarea o sencillamente mayor cantidad de experiencia. El hecho de que todo esto no pueda ser cuantificado y clasificado en estados secuenciales de progreso es absolutamente irrelevante (Hodson, 1992a).
En España no tenemos ni una larga tradición ni una gran experiencia en el trabajo práctico para la enseñanza de las ciencias. Los nuevos currículos no universitarios tampoco son demasiado explícitos en este aspecto. Los recién estrenados planes de estudios para las carreras universitarias de ciencias parece que sí han apostado por proporcionar al trabajo práctico un papel más relevante que el que tenía anteriormente, aumentando tanto el número de horas de enseñanza práctica como la cantidad de materias con un carácter eminentemente experimental. Este hecho, si además de sobre el papel llega a materializarse en la práctica educativa, hace concebir esperanzas de que los futuros profesores de ciencias de enseñanza secundaria y bachilierato obtengan de su formación inicial una mayor sensibilidad por el trabajo práctico y un mayor nivel de maestría en las tareas de investigación científjca. Además, sería muy interesante el establecimiento de colaboraciones entre los profesores de ciencias en activo y los investigadores de las universidades, para así proporcionar a los primeros la oportunidad de conocer lo que significa hacer ciencia y mejorar su condición de investigadores expertos, característica necesaria para introducir la concepción holista de las ciencias en la instrucción científica.

Igualmente, habria que proporcionar en la formación inicial de estos profesores la oportunidad para que desarrollen sus capacidades de emitir juicios profesionales y tener confianza en ellos, ya que el trabajo práctico enfocado como investigación sólo puede ser evaluado correctamente de una forma global, holista, en la gue las técnicas cualitativas de evaluación y los estudios de casos concretos deben jugar un papel importante.

Para el tratamiento de las ciencias en la educación primaria en España, el panorama que se presenta es sombrío, ya que los nuevos planes de estudio diseñados para la formación inicial de los maestros de educación infantil y primaria apenas contemplan la instrucción en ciencias. Ésta es una carencia muy grave que puede acarrear grandes problemas en un futuro próximo y no sólo sobre el trabajo práctico a realizar en esas cdades tempranas, sino en toda la educación que sobre ciencias se realiza en esos niveles educativos. España es el país que tiene el porcentaje menor de titulados en ciencias e ingenierías de todos los pertenecientes a la OCDF; (un $13,6 \%$, frente a valores como el máximo de Alemania, del $70 \%$, el $30 \%$ en los Estados Unidos de América o el $45 \%$ en Italia; NSF, 1993; UNESCO, 1993). La reforma de las enseñanzas que estamos llevando a cabo parece ignorar este punto y que las ciencias forman parte integral de la cultura occidental actual en un nivel equiparable al que ocupan las humanidades. Esta negligencia con que se tratan las ciencias en estos planes nuevos (Jiménez y Sanmartí, 1995) no nos hace ser demasiado optimistas sobre la consideración del trabajo práctico que depararán los nuevos currículos, y no debemos olvidar que una inadecuada educación científica en los niveles preuniversitarios quizás sea un factor capaz de explicar por sí solo la escasa motivación de los estudiantes españoles por las carreras universitarias de ciencias, y el consiguiente bajo porcentaje de esta clase de licenciados en nuestro país. 
Para terminar, únicamente cabe repetir el final endémico de todos los informes que tratan el trabajo práctico en la enseñanza de las ciencias: se precisa mucha más y mejor investigación sobre este tema que es considerado crucial por todos los que estamos envueltos de una forma u otra en transmitir a las nuevas generaciones la herencia cultural que denominamos ciencias.

\section{REFERENCIAS BIBLIOGRÁFICAS}

ABRAMS, E. y WANDERSEE,J.H.(1995a). How does biological knowledge grow? A study of life scientists' research practices. Journal of Research in Science Education, 32, pp.649-663.

ABRAMS, E. y WANDERSEE, J.H. (1995b). How to infuse actual scientific research practices into science classroom instruction. International Journal of Science Education, 17, pp. 683-694.

ANDERSON, O.R. (1976). The experience of science: a new perspective for laboratory teaching. Nueva York: Teachers College Press.

ASTOLFI, J.P. (1994), El trabajo didáctico de los obstáculos, en el corazón de los aprendizajes científicos. Enseñanza de las Ciencias, 12, pp. 206-216.

ASTOLFI, I.P. y PETERFALVI, B. (1993). Obstacles et construction de situations didactiques en sciences expérimentales. Aster, 3, pp. 73-110.

ATKINSON, E.P. (1990). Learning scientific knowledge in the student laboratory, en The student laboratory and the science curriculum, editado por Elizabeth Hegarty-Hazel. Londres: Routledge.

BL.ACK, P.J. (1993). Formative and summative assessment by teachers. Studies in Science Education, 21, pp. 49-97.

BLACK, P.J. (1995). Curriculum and assessment in science education: the policy interface. International Journal of Science Education, 17, pp. 453-469.

BIISS, J. (1990). Student's reactions to undergraduate science: laboratory and project work, en The siudent laboratory and the science curriculum, editado por Elizabeth HegartyHazel. Londres: Routledge.

BLOOM,B.S.(1956). Taxonomia de losobjetivos de la educación: la clasificación de las metas educacionales. Alcoy: Marfil (1973).

BOUD, D.J., DUNN, J. y HEGARTY-HAZEL, E. (1986). Teaching in laboratories. Guilford: The Society for Research into Higher Education \& NFER-NELSON.

BOUD, D.J., DUNN, J., KENNEDY, T. y THORLEY, R. (1980). The aims of science laboratory courses: a survey of students, graduates and practising scientists. European Journal of Science Education, 2, pp. 415-428.

\section{AGRADECIMIENTOS}

Uno de los autores (O.B.) quicre agradecer a Ia DGICYT la financiación recibida para esta investigación (PR95-231), y a las personas del Centre for Applied Research in Educution (University of East Anglia, Norwich, Reino Unido), su amable acogida.

BRICKHOUSE, N.W., STANLEY, W.B. y WHITSON, J.A. (1993). Practical reasoning and science education: implications for theory and practice. Science and Education, 2, pp. 363-375.

BRYCE, T.G.K. y ROBERTSON, I.J. (1985). What can they do? A review of practical assessment in science. Studies in Science Education, 12, pp. 1-24.

BRYCE, T.G.K., MCCALI, J., MACGREGOR, J., WFSTON, R.A.J. y ROBERTSON, I.J. (1983). Techniques for the assessment of practical skills (TAPS) in foundation science. Londres: Heinemann Educational Books.

BUCHAN, A.S. (1992). Practical assessment in GCSE science. The diversity of the examination groups practices. School Science Review, 73(265) pp. 19-28.

BUCHAN, A.S. y JENKINS, E.W.(1992). The internal asscssment of practical skills in science in England and Wales 19601991: Some issues in historical perspective. International Journal of Science Education, 14, pp. 367-380.

CAWTHRON, E.R. y ROWELL, J.A. (1978). Epistemology and science education. Studies in Science Education, 5, pp. 51-59.

CLACKSON, S.G. y WRIGHT, D.K. (1992). An appraisal of practical work in science education. School Science Review, 74(266), pp. 39-42.

DES (Department of Education and Science) (1985). Science at age IS. Science Report for Teachers. Londres: Her Majesty's Stationery Office.

DESAUTELS, J., LAROCHELLE, M., GAGNÉ, B. y RUEL, F. (1993). La formation à l'enseignement des sciences: Ie virage épistémologique. Didaskalia, 1, pp. 49-67.

DORAN, R.I., LAWRENZ, F, y HEGELSON, S. (1994). Research on assessment in science, en Handbook of Re search on Science Teaching and Learning, editado por Dorothy L. Gabel. Nueva York: Macmillan.

DUGGAN, S. y GOTT, R. (1995). The place of investigations in practical work in the UK National Curriculum for Science. International Journal of Science Education, 17, pp. 137-147.

DRIVER, R. (1985). Beyond appearance: The conservation of matter under chemical and physical transformations, en 
Driver, R., Guesne, E. y Tiberghien, A. (eds.), Children's ideas in science. Milton Keynes: Open University Press. Trad. cast. (1989), Las ideas científicas en la infancia y en la adolescencia. Madrid: Morata/MEC.

EDWARDS, J. y POWER, C. (1990). Role of laboratory work in a national junior secondary science project: Australian Science Education Project (ASEP), en The student laboratory and the science curriculum, editado por Elizabeth HegartyHazel. Londres: Routledge.

FENSHAM, P.J. (1990). Practical work and the laboratory in Science for All, en The student laboratory and the science curriculum, editado por Elizabeth Hegarty-Hazel. Londres: Routledge.

FRASER, J.B., GIDDINS, G.J. y McROBBIE, C.J. (1995). Evolution and validation of a personal form of an instrument for assessing science laboratory classroom environments. Journal of Research of Science Teaching 32, pp. 399-422.

FRIEDLER, Y. y TAMIR, P. (1986). Teaching basic concepts of scientific research to high school students. Journal of Biological Education, 20, pp. 263-269.

FRIEDLER, Y, y TAMIR, P. (1990). Life in science laboratory classroom at secondary level, en The student laboratory and the science curriculum, editado por Elizabeth HegartyHazel. Londres: Routledge.

GARDNER, P. y GAULD, C. (1990). Labwork and students attitudes, en The student laboratory and the science curriculum, editado por Elizabeth Hegarty-Hazel. Londres: Routledge.

GARRET, R.M. y ROBERTS, T.F. ( 1982). Demostration versus small group practical work in science education. A critical review of studies since 1900. Studies in Science Education, 9, pp. 109-146.

GEF, B. y CLACKSON, S.G. (1992). The origin of practical work in the English school science curriculum. School Science Review, 73 (265), pp. 79-83.

GIL, D. (1993). Contribución de la historia y la filosofía de las ciencias al desarrollo de un modelo de enseñanza-aprendizaje como investigación. Enseñanza de las Ciencias, 11 pp. 197 212 .

GUNSTONE, R.F. (1991). Reconstructing theory from practical experience, en Practical science. The role and reality of practical work in school science, editado por Brian, $\mathrm{E}$. Woolnough. Milton Keynes: Open University Press.

HEAD, J. (1982). What can psychology contribute to science education? School Science Review, 63(225), pp. 631-642.

HEGARTY-HAZEL, E. (1990a). Learning technical skills in the student laboratory, en The student laboratory and the science curriculum, editado por Elizabeth Hegarty-Hazel. Londres: Routledge.

HEGARTY-HAZEL, E. (1990b). Life in science laboratory classroom at tertiary level, en The st udent laboratory and the science curriculum, editado por Elizabeth Hegarty-Hazel. Londres: Routledge.

HODSON, D. (1988). Experiments in science and science teaching. Educational Philosophy and Theory, 20, pp. 53-66.

HODSON, D. (1990). A critical look at practical work in school science. School Science Review, 71 (256), pp. 33-40.
HODSON, D. (1992a). Assessment of practical work. Some considerations in philosophy of science. Science and Education, 1, pp. 115-144.

HODSON, D. (1992b). Redefining and reorienting practical work in school science. School Science Review, 73(264), pp. 65-78.

HODSON, D. (1993). Re-thinking old ways: towards a more critical approach to practical work in school science. Studies in Science Education, 22, pp. 85-142.

HODSON, D. (1994). Hacia un enfoque más crítico del trabajo de laboratorio. Enseñanza de las Ciencias, 12 (3), pp. 299-313.

HOFSTEIN; A. y LUNETTA, V.N. (1982). The role of laboratory in science teaching: neglected aspects of research. Review of Educational Research, 52, pp. 201-217.

IGELSRUD, D. y LEONARD, W.H. (1988). What research says about biology laboratory instruction. The American Biology Teacher, 50, pp. 303-306.

JENKINS, E.W. (1995a). Central policy and teacher response? Scientific investigation in the national curriculum of England and Wales. International Journal of Science Education, 17, pp. 471-480.

JENKINS, E.W. (1995b). When is a policy not a policy? School-based assessment of practical science at $16+$. International Journal of Science Education, 17, pp. 555563.

JIMÉNEZ, M.P. y SANMARTÍ, N. (1995). The development of a new science curriculum for secondary school in Spain: opportunities for change. International Journal of Science Education, 17, pp. 425-439.

JOHNSON, S. (1989). National Assessment: the Assessment of Performance Unit (APU) science approach. Londres: Her Majesty's Stationery Office.

KERR, J.F. (1963). Practical work in school science. Leicester: Leicester Universily Press.

KIMBELL, R. (1991). Tackling technological tasks, en Practical science. The role and reality of practical work in school science, editado por Brian E. Woolnough. Milton Keynes: Open University Press.

KIRSCHNER, P.A. (1992). Epistemology, practical work and academic skills in science education. Science and Education, ], pp. 273-299.

KIRSCHNER, P.A., MEESTER, M., MIDDLEBEEK, E. y HERMANS, H. (1993). Agreement between students expectations, experiences and actual objectives of practicals in the natural sciences at the OpenUniversity of the Netherlands. International Journal of Science Education, 15, pp. 175197.

KOPFLER, L.E. (1990). Learning scientific enquiry in the student laboratory, en The student laboratory and the science curriculum, editado por Elizabeth Hegarty-Hazel. Londres: Routledge.

KYLE, Jr., W.C., PENICK, J.E. y SHYMANSKY, J.A. (1979). Assessing and analizing the performance of students in college science laboratories. Joumal of Research in Science Teaching, 16, pp. $545-551$. 
LAYTON, D. (1990). Student laboratory practice and the history and philosophy of science, en The student laboratory and the science curriculum, editado por Elizabeth HegartyHazel. Londres: Routledge.

I.A7AROVITZ, R. y TAMIR, P. (1994). Research on using laboratory instruction in science, en Handbook of Research on Science Teaching and Leaming, editado por Dorothy $L$. Gabel. Nueva York: Macmillan.

I.EDFRMAN, N.G. (1992). Students' and teachers' conceptions of the nature of science: a revicw of the research. Journal of Research in Science Teaching, 29, pp. 331-359.

LEWIS, R.W. (1982). Theories, structure, teaching, and learning. BioScience, 32, pp. 734-737.

LEWIS, R.W. (1988). Biology: A hypothetico-deductive science. The American Biology Teacher, 50, pp. 362-366.

I.OCK, R. (1988). A history of practical work in school science and its assessment, 1860-1986. School Science Review, $70(250)$, pp. 115-119.

LYNCH,P.P. (1987). Laboratory work in schools and universities: Structures and strategies still largely unexplored. The Australian Sciences Teacher Joumal, 32, pp. 31-39.

I.YNCH, P.P. y NDYETABURA, V.I. (1984). Students' attitudes to school practical work in Tasmanian schools. The Australian Science Teachers Journal, 32, pp. 31-39.

MAYER, W.V. (1986). Biology education in the United States during the Twentieth Century. The Quarterly Review of Biology, 61, pp. 481-507.

MEESTER, M.A.M. y MASKILL, R.(1993). First year practical classes in undergraduate chemistry courses in England and Wales. Londres: The Royal Society of Chemistry.

MEFSTER, M.A.M. y MASKILL, R. (1994). Second year practical classes in undergraduate chemistry courses in England and Wales. Londres: The Royal Society of Chemistry.

MEESTER, M.A.M. y MASKILL,R.(1995a). First-yearchemistry practicals at universities in England and Wales: aims and the scientific level of the experiments. International Journal of Science Education, 17, pp. 575-588.

MEESTER, M.A.M. y MASKILL, R. (1995b). First-year chemistry practicals at universities in England and Wales: organizational and teaching aspects. International Journal of Science Education, 17, pp. 705-719.

MEICISTRY, Y. (1993). The impact of science currictila on students views about the nature of science. Journal of Research in Science Teaching, 39, pp. 429-443.

MIGUENS, M. y GARRET, R.M. (1991). Prácticas en la enseñanza de las ciencias. Problemas y posibilidades. Enseñanza de las Ciencias, 9, pp. 229-236.

MIILAR, R. (1991). A means to an end: The role of processes in science education, en Practical science. The role and reality of practical work in school science, editado por Brian E. Woolnough. Milton Keynes: Open University Press

MIL,I,AR, R. y DRIVER, R. (1987). Beyond processes. Studies in Science Education, 14, pp. 33-62.
MOYER, A.E. (1976). Edwin Hall and the emergence of the laboratory in teaching physics. The Physics Teacher, 14, pp. 96-103.

NORRIS, S.P. (1995). Learning to live with scientific expertise: toward a theory of intellectual communalism for guiding science teaching. Science Education, 79, 201-217. Ver también: Grandy, R.E. (1995). Fxpertise and the ctitical nonexpert (comment on Norris), Science Education, 79, pp. 219-221; y Norris, S.P. (1995). Reaching the «Hardwig limit»: nonscientists' ability to sniff out scientific bias and to judge scientific research methods (response to Grandy), science Education, 79, pp. 223-227.

NOVAK, J.D. (1978). An alternative to Piagetian psychology for science and mathematics education. Studies in Science Education, 5, pp. I-30.

NRC (National Research Council)(1990). Fulfilling the promise: biology education in the Nation's schools. Washington, D.C.: National Academy Press.

NSF (National Science Foundation) (1993). Scienve and engineering indicators. Washington D.F.: National Science Foundation.

PICKERING, M. (1980). Are lab courses a waste of time? Chronicle of Higher Education, 19, pp. 44-50.

PRAIA, J. y CACHAPUZ, F. (1994). Un análisis de las concepciones acerca de la naturaleza del conocimiento científico de los profesores portugueses de la enseñanza secundaria. Enseñanza de las Ciencias, 12, pp. 350-354.

QUAI.TER, A., STRANG, J., SWATTON, P. y TAYLOR, R. (1990). Exploration. A way of learning science. Oxford: Blackwell.

REIF, F. y ST. JOHN, M. (1979). Teaching physicists thinking skills in the laboratory. American Journal of Physics, 47, pp. $750-757$.

SCIENCE (informe de varios autores) (1996). Science Fducation: European Universities in transition. Srience, 271(5249), pp. $681-701$

SHULMAN, L.E. y TAMIR, P. (1973). Research on teaching in the Natural Sciences, en Second Handbook of Rerearch on Teaching, editado por Robert, M.W. Travers. Chicago: Rand McNally College Publishing.

SMOLICZ, J.J. y NUNAN, E.E. (1975). The philosophical and sociological foundations of science education: the demythologizing of school science. Studies in Science Education, 2, pp. 101-143.

STAKE, R.E. y EASLEY, J.A., Jr, (dirs.) (1978). Case Studies in Science Education. Urbana-Champaign: University of Illinois.

STAWINSKI, W. (1986). Research into the effectiveness of student experiments in biology teaching. European Journul of Science Education, 8, pp. 213-224.

SUNDBERG, M.D. y ARMSTRONG, J.E. (1993). The status of laboratory instruction for introductory biology in US universities. The American Biology Teacher, 55, pp. 144I 46 .

TAMIR, P. (1974). An inquiry oriented laboratory examination. Journal of Educational Measurement, 11, pp. 25-33. 
TAMIR, P. (1977). How are the laboratories used? Journal of Research in Science Teaching, 14, pp. 311-316.

TAMIR, P. y GARCIA, M.P.(1992). Characteristics of laboratory exercises included in science textbooks in Catalonia (Spain). International Journal of Science Education, 14, pp. 381392.

TAMIR, P. y LUNETTA, V.N. (1978). An analysis of laboratory activities in the BSCS Yellow Version. The American Biology Teacher, 40 , pp. 353-357.

TAMIR, P. y LUNETTA, V.N. (1981). Inquiry related tasks in high school science laboratory handbooks. Science Education, 65 , pp. 477-484

THIJS, G.D. y BOSCH, G.M. (1995). Cognitive effects of science experiments focusing in students 'preconceptions of force: a comparison of demonstrations and small group practices. International Journal of Science Education, 17 , pp. 31 I-323.

TOBIN, K. (1986). Secondary science laboratory activities European Journal of Science Education, 8, pp. 199-211.

TOBIN, K. y GALLAGHER, IJ. (1987). What happens in high school science classrooms? Journal of Curriculum Studies, 19, pp. 549-560.

TOH, K.A. y WOOLNOUGH, B.E. (1990). Assessing, through reporting, the outcomes of scientific investigations. Educational Research, 32, pp. 59-65

TOH, K.A. y WOOLNOUGH, B.E. (1993). Middle school students' achievement in laboratory investigations: explicit versus tacit knowledge. Journal of Research in Science Teaching, 30, pp. 445-457. Ver también: Ross, J.A. (1995). A comment on explicit versus tacit knowledge: student achievement in laboratory investigations. Journal of Research in Science Teaching, 32, pp. 997-998 y Toh, K.A. y Woolnough, B.E. (1995). Explicit versus tacit knowledge in laboratoty investigations: a reply to Ross. Journal of Research in Science Teaching, 32, pp. 999-1001.

TOOTHACKER, W.S. (1983). A critical look at introductory laboratory instruction. American Journal of Physics, 51, pp. 516-520.
TREMLETT, R. (1972). An investigation into the development of a programme of practical work for undergraduates in chemistry, 2 vol. Tesis doctoral, University of East Anglia, Norwich (UK)

UNESCO (1993). World science report. París: UNESCO.

WATSON, J.R., PRIETO, T. y DILLON, J.S. (1995). The effect of practical work on students understanding of combustion. Journal of Research in Science Teaching, 32, pp. 487-502.

WATSON, J.R. y PRIETO, T. (1994). Secondary science in England and Spain. Education in Chemistry, 31, pp. 40-41.

WATTS, M. (1991). The science of problem solving. A practical guide for science teachers. Londres: Cassell Educational.

WEINBURGH, M.H. y ENGLEHARD JR., G. (1994). Gender prior academic performance and beliefs as predictors of attitudes toward biology laboratory experiencies. School Science and Mathematics, 94, pp. 118-123.

WELLINGTON, J. (ed.) (1989). Skills and processes in science education. A critical analysis. Londres: Routledge.

WHITE, R.T. (1979). Relevance of practical work to the comprehension of physics. Physics Education, 14, pp. 384-387

WHITE, R.T. (1991). Episodes and the purpose and conduct of practical work, en Practical science. The role and reality of practical work in school science, editado por Brian E. Woolnough. Milton Keynes: Open University Press.

WOLPERT, L. (1993). The unnatural nature of science. Londres: Faber \& Faber.

WOOLNOUGH, B.E. (1991). Practical science as a holistic activity, en Practical science. The role and reality of practical work in school science, editado por Brian E. Woolnough. Milton Keynes: Open University Press.

WOOLNOUGH, B.E. y ALLSOP, T. (1985). Practical work in science. Cambridge: Cambridge University Press.

WOOLNOUGH, B.E. y TOH, K,A. (1990). Altemative approaches to assessment of practical work in science. School Science Review, 71(256), pp. 127-131

[Articulo recibido en febrero de 1996 y aceptado en marzo de 1996.] 
. 